\title{
The Boussinesq system with mixed nonsmooth boundary data*
}

\section{Le système de Boussinesq à données limites mixtes peu regulières}

\author{
E. J. Villamizar-Roa ${ }^{\mathrm{a}}$ M. A. Rodríguez-Bellido ${ }^{\mathrm{b}}$ M. A. Rojas-Medar ${ }^{\mathrm{c}}$ \\ ${ }^{a}$ Escuela de Matemáticas, Universidad Industrial de Santander, A.A. 678, Bucaramanga-Santander, Colombia \\ ${ }^{\mathrm{b}}$ Dpto. de Ecuaciones Diferenciales y Análisis Numérico, Universidad de Sevilla, \\ Apto. 1160, 41080 Sevilla, Spain \\ ${ }^{\mathrm{c}}$ IMECC-UNICAMP, CP 6065, 13083-970, Campinas-SP, Brazil
}

\begin{abstract}
We treat the stationary Boussinesq system with non-smooth mixed boundary conditions for the temperature, and non-smooth Dirichlet boundary condition for the velocity. We prove the existence, the continuous dependence of the solution with respect to the data and the uniqueness of the very weak solution. To cite this article: E. J. Villamizar-Roa, M. A. Rodríguez-Bellido \& M. A. Rojas-Medar, C. R. Acad. Sci. Paris, Ser. I 336 (2003).
\end{abstract}

\section{Résumé}

On traite le système de Boussinesq stationnaire à données au bord mixtes peu régulières pour la température, et donnée au bord Dirichlet peu régulière pour la vitesse. On montre l'existence, la dépendance continue de la solution par rapport aux données et l'unicité de solution très faible pour ce système. Pour citer cet article : E. J. Villamizar-Roa, M. A. Rodríguez-Bellido E3 M. A. Rojas-Medar, C. R. Acad. Sci. Paris, Ser. I 336 (2003).

\section{Version française abrégée}

On considère le système de Boussinesq avec conditions aux limites peu régulières, mixtes DirichletNeumnann pour la température et Dirichlet pour la vitesse. On introduit les solutions très faibles, et on montre l'existence et dépendance continue de ce type de solution par rapport aux données extérieures et les conditions aux limites. Concretement, les résultats qu'on prouve sont les suivants :

\footnotetext{
* The first author is supported by Universidad Industrial de Santander and COLCIENCIAS-Colombia, Project COLCIENCIAS-BID III etapa. The second and third authors have been partially supported by D.G.E.S. \& M.C. y T.(Spain), Projet BFM2003-06446-C02-01. The third author has been partially supported by CNPq-Brazil, grant No 301354/03-0

Email addresses: jvillami@uis.edu.co (E. J. Villamizar-Roa), angeles@us.es (M. A. Rodríguez-Bellido), marko@ime.unicamp.br (M. A. Rojas-Medar).
} 
Théorème 0.1 (Existence) Si la viscosité cinématique $\nu$ est assez grande par rapport à $|\boldsymbol{f}|_{3},|\xi|_{L^{2}\left(\Gamma_{1}\right)}$ et $|\zeta|_{H^{-1}\left(\Gamma_{2}\right)}$, alors il existe une solution très faible du problème (2).

Théorème 0.2 (Dépendance continue) Soient $\left(\boldsymbol{u}_{i}, \theta_{i}\right), i=1,2$ deux solutions très faibles du problème (2) pour les forces externes $\boldsymbol{f}=\boldsymbol{f}_{i} \in \mathbf{L}^{3}(\Omega), h=h_{i} \in L^{2}(\Omega), i=1,2$ et les données au bord $\mathbf{g}=\mathbf{g}_{i} \in$ $\mathbf{L}^{2}(\Gamma), \zeta=\zeta_{i} \in H^{-1 / 2}\left(\Gamma_{2}\right), \xi=\xi_{i} \in L^{2}\left(\Gamma_{1}\right), i=1,2$, respectivement. Alors, il existe une constante $\nu^{*}>0$ telle que pour toute $\nu \geq \nu^{*}$,

$$
\begin{aligned}
\left|\boldsymbol{u}_{1}-\boldsymbol{u}_{2}\right|_{3}+\left|\theta_{1}-\theta_{2}\right|_{2} & \leq c\left\{\left|\boldsymbol{f}_{1}-\boldsymbol{f}_{2}\right|_{3}+\left|h_{1}-h_{2}\right|_{2}+\left|\xi_{1}-\xi_{2}\right|_{L^{2}\left(\Gamma_{1}\right)}+\left|\zeta_{1}-\zeta_{2}\right|_{H^{-1 / 2}\left(\Gamma_{2}\right)}\right. \\
& \left.+\left|\mathbf{g}_{1}^{\epsilon}-\mathbf{g}_{2}^{\epsilon}\right|_{H^{1}(\Gamma)}+\left|\left(\mathbf{g}_{1}-\mathbf{g}_{1}^{\epsilon}\right)-\left(\mathbf{g}_{2}-\mathbf{g}_{2}^{\epsilon}\right)\right|_{L^{2}(\Gamma)}\right\}
\end{aligned}
$$

où $\mathbf{g}_{i}^{\epsilon}$ sont des fonctions suffisamment proches de $\mathbf{g}_{i}, i=1,2$, dans la norme $\mathbf{L}^{2}(\Gamma)$ et la constante $c$ ne dépend que des données du problème et de $\Omega$. En particulier, pour $\nu \geq \nu^{*}$, la solution de (2) est unique.

\section{Introduction}

We consider the following Boussinesq System [1], with nonsmooth boundary data:

$$
\left\{\begin{aligned}
-\nu \Delta \mathbf{u}+(\mathbf{u} \cdot \nabla) \mathbf{u}+\nabla p & =\beta \mathbf{f} \theta \text { in } \Omega, & \nabla \cdot \mathbf{u}=0 \text { in } \Omega, & \mathbf{u}=\mathbf{g} \text { on } \Gamma \\
-\chi \Delta \theta+(\mathbf{u} \cdot \nabla) \theta=h \text { in } \Omega, & \partial_{\mathbf{n}} \theta=\zeta \text { on } \Gamma_{2}, & \theta & =\xi \text { on } \Gamma_{1}
\end{aligned}\right.
$$

where the unknowns of the problem are $\mathbf{u}(\mathbf{x}) \in \mathbb{R}^{3}$ the fluid velocity, $p(\mathbf{x}) \in \mathbb{R}$ the pressure and $\theta(\mathbf{x}) \in \mathbb{R}$ the temperature. The data are $\Gamma=\Gamma_{1} \cup \Gamma_{2}$ the boundary of $\Omega, h(\mathbf{x}) \in \mathbb{R}$ the reference temperature, $\mathbf{f}(\mathbf{x}) \in$ $\mathbb{R}^{3}$ the gravitational field at $\mathbf{x}$, and $\nu, \beta, \chi>0$ that represent the kinematic viscosity, the coefficient of volume expansion and thermal conductance, respectively. Without loss of generality, we have taken the density of fluid to be constant and equal to one. We consider the following two types of domains $\Omega \subseteq \mathbb{R}^{3}$ : CASE 1. As in [3], we consider a bounded subset $\Omega=\left\{(y, \mathbf{z}) \in \mathbb{R} \times \mathbb{R}^{2}: \mathbf{z} \in P, \Phi_{0}(\mathbf{z})<y<\Phi_{1}(\mathbf{z})\right\}$, where $P$ is a plane curvilinear polygon with $C^{2}$ sides, without cusp ends, $\Phi_{0}(\mathbf{z})$ and $\Phi_{1}(\mathbf{z})$ are $C^{2}(\bar{P})$ functions, such that for all $\mathbf{z} \in \bar{P}, \Phi_{0}(\mathbf{z})<\Phi_{1}(\mathbf{z})$. We denote by $\Gamma$ the boundary of $\Omega$ and we consider the following partition of $\Gamma$ :

$$
\Gamma_{2}=\left\{(y, \mathbf{z}) \in \mathbb{R} \times \mathbb{R}^{2}: \mathbf{z} \in \partial P, \Phi_{0}(\mathbf{z})<y<\Phi_{1}(\mathbf{z})\right\}, \Gamma_{1}=\cup_{i=0,1}\left\{(y, \mathbf{z}) \in \mathbb{R} \times \mathbb{R}^{2}: \mathbf{z} \in P, y=\Phi_{i}(\mathbf{z})\right\}
$$

We denote by $\omega$ the largest inner angle of $P$ and $\phi$ the supremum of the dihedral angles between $\Gamma_{2}$ (side of the cylinder) and $\Gamma_{0}$ or $\Gamma_{1}$ (bases of the cylinder). We suppose $0<\phi \leq \pi / 2$ and $\omega<\pi$.

CASE 2. The boundary $\Gamma$ of $\Omega$ is of $C^{2}$ class and it is divided into two parts, $\Gamma=\Gamma_{1} \cup \Gamma_{2}$ with measure of $\Gamma_{1}$ non zero and $\overline{\Gamma_{1}} \cap \overline{\Gamma_{2}}=\emptyset$. A typical example is $\Omega=Q_{1} \backslash Q_{2}, \bar{Q}_{2} \subset Q_{1}$ being $Q_{1}, Q_{2}$ balls of radius $r_{1}, r_{2}\left(r_{1}>r_{2}\right)$ respectively, which can represent the case of an obstacle within a fluid.

As usual $\left(L^{p}(\Omega),|\cdot|_{p}\right)$, with $1 \leq p \leq+\infty$ and $\left(W^{k, p},\|\cdot\|_{k, p}\right)$ are the usual Sobolev spaces. In particular $H^{k}(\Omega)=W^{k, 2}(\Omega)$ with the norm $\|\cdot\|_{k}=\|\cdot\|_{k, 2}$. By $(\cdot, \cdot)$, we represent the inner product in $L^{2}(\Omega)$. We denote $H_{\Gamma_{1}}^{1}(\Omega):=\left\{u \in H^{1}(\Omega): u \equiv 0\right.$ on $\left.\Gamma_{1}\right\}$ and $\mathbf{V}$ the closure of $\left\{\mathbf{u} \in C_{0}^{\infty}: \nabla \cdot \mathbf{u}=0\right.$ in $\left.\Omega\right\}$ in the norm of $H^{1}(\Omega)$, being $((\cdot, \cdot))$ and $\|\cdot\|$ the corresponding inner product and norm. We consider the system (2) with nonhomogeneous boundary conditions for $\mathbf{u}$ and $\theta$; we only assume $\zeta \in H^{-1 / 2}\left(\Gamma_{2}\right), \xi \in L^{2}\left(\Gamma_{1}\right)$ and $\mathbf{g} \in L^{2}(\Gamma)^{3}$ with $\int_{\Gamma} \mathbf{g} \cdot \mathbf{n}=0, \mathbf{g} \cdot \mathbf{n}=0$ on $\Gamma_{2}$, where $\mathbf{n}$ denotes the unit outward normal of $\Gamma$. Some authors (see for instance [11]) considered mixed boundary conditions for $\theta$ and boundary Dirichlet conditions for $\mathbf{u}$ but their works were done for regular functions $\mathbf{g}, \zeta$ and $\xi$, so these functions can be extended to the interior of the domain $\Omega$. This allows one to use standard arguments in order to obtain 
the existence of weak solutions. However, when the boundary data are not regular, that is, when the usual trace theorems cannot be used, the solvability of (2) has not been investigated, as far as we know. In this work, we only assume $\mathrm{g} \in L^{2}(\Gamma), \xi \in L^{2}\left(\Gamma_{1}\right)$ and $\zeta \in H^{-1 / 2}\left(\Gamma_{2}\right)$, and prove the existence of a very weak solution (see Definition 1.1 below), that is a natural extension of the weak solution and it was used by Conca [2] for the Stokes problem. These results were extended in [9] to the Navier-Stokes equations, and in [12] to the Boussinesq equations with Dirichlet boundary conditions for $\theta$ and $\mathbf{u}$.

In order to have a well-posed elliptic problem with mixed boundary data for $\theta$, we need to impose some hypothesis over the domain. This is a quite interesting question which has been studied by M. Dauge in $[3,4]$. The elliptic problem for the temperature is coupled with a Navier-Stokes system for the velocity, which must be solved in a nonsmooth domain. We recall that the domains considered in [9] were of $C^{1,1}$ class. Our main results are:

Definition 1.1 A triple $(\boldsymbol{u}, \theta, p)$ in $\mathbf{L}^{3}(\Omega) \times L^{2}(\Omega) \times W^{-1,3}(\Omega)$ is a very weak solution of problem (2) if

$$
\begin{aligned}
& \int_{\Omega}(-\nu \boldsymbol{u} \cdot \Delta \Phi-(\boldsymbol{u} \cdot \nabla) \Phi \cdot \boldsymbol{u}) d \mathbf{x}-\langle p, \nabla \cdot \Phi\rangle_{W^{-1,3}(\Omega), W^{1,3 / 2}(\Omega)}=\beta \int_{\Omega} \theta \boldsymbol{f} \cdot \Phi d \mathbf{x}-\nu \int_{\Gamma} \boldsymbol{g} \cdot \partial_{\mathbf{n}} \Phi d s, \\
& \int_{\Omega} \boldsymbol{u} \cdot \nabla \tau d \mathbf{x}=\int_{\Gamma}^{\Omega}(\boldsymbol{g} \cdot \mathbf{n}) \tau d s \\
& -\chi(\theta, \Delta \psi)-(\boldsymbol{u} \cdot \nabla \psi, \theta)=(h, \psi)+\chi\langle\zeta, \psi\rangle_{H^{-1 / 2}\left(\Gamma_{2}\right), H^{1 / 2}\left(\Gamma_{2}\right)}-\chi \int_{\Gamma_{1}} \xi \partial_{\mathbf{n}} \psi d s,
\end{aligned}
$$

$\forall \Phi \in \mathbf{W}^{2,3 / 2}(\Omega) \cap \mathbf{W}_{0}^{1,3 / 2}(\Omega), \forall \tau \in W^{1,3 / 2}(\Omega)$ with $\int_{\Omega} \tau d \mathbf{x}=0$ and $\forall \psi \in H^{2}(\Omega) \cap H_{\Gamma_{1}}^{1}(\Omega)$ with $\left.\partial_{\mathbf{n}} \psi\right|_{\Gamma_{2}} \equiv 0$. Theorem 1.2 (Existence) If $\nu$ is large enough with respect to the norms $|\boldsymbol{f}|_{3},|\xi|_{L^{2}\left(\Gamma_{2}\right)}$ and $|\zeta|_{H^{-1 / 2}\left(\Gamma_{2}\right)}$, then there exists a very weak solution of the problem (2) in the sense of the above definition.

Theorem 1.3 (Continuous Dependence) Let $\left(\boldsymbol{u}_{i}, \theta_{i}\right), i=1,2$ be very weak solutions of problem (2) corresponding to the external forces $\boldsymbol{f}=\boldsymbol{f}_{i} \in \mathbf{L}^{3}(\Omega), h=h_{i} \in L^{2}(\Omega), i=1,2$ and boundary data $\mathbf{g}=\mathbf{g}_{i} \in \mathbf{L}^{2}(\Gamma), \zeta=\zeta_{i} \in H^{-1 / 2}\left(\Gamma_{2}\right), \xi=\xi_{i} \in L^{2}\left(\Gamma_{1}\right), i=1,2$, respectively. Then, there exists a constant $\nu^{*}>0$ such that for all $\nu \geq \nu^{*}$, (1) is verified. In particular, for $\nu \geq \nu^{*}$, the solution of (2) is unique.

\section{Problem in $\theta$}

Problem 1. Consider $h \in L^{2}(\Omega), \xi \in L^{2}\left(\Gamma_{1}\right), \zeta \in H^{-1 / 2}\left(\Gamma_{2}\right)$ and choose (see $\left.\S 3\right) \mathbf{u} \in \mathbf{L}^{3}(\Omega)$ with $\nabla \cdot \mathbf{u}=0$ (in the weak sense), splitting $\mathbf{u}$ into $\mathbf{u}^{\epsilon}+\mathbf{v}^{\epsilon}$, being $\mathbf{u}^{\epsilon} \in \mathbf{H}^{1}(\Omega)$ the regular part and $\mathbf{v}^{\epsilon} \in \mathbf{L}^{3}(\Omega)$ the irregular part with $\left|\mathbf{v}^{\epsilon}\right|_{3}$ small enough. First, we want to find $\theta$ in $L^{2}(\Omega)$ such that $(3)_{3}$ holds.

Lemma 2.1 There exists a unique solution of Problem 1.

Outline of the proof: For the data $b \in L^{2}(\Omega)$, we consider the weak solution $\psi \in H_{\Gamma_{1}}^{1}(\Omega)$ of

$$
-\chi \Delta \psi-(\mathbf{u} \cdot \nabla) \psi=b \text { in } \Omega, \quad \psi=0 \text { on } \Gamma_{1}, \quad \partial_{\mathbf{n}} \psi=0 \text { on } \Gamma_{2} .
$$

There exists a constant $c=c(\Omega)$ such that $\chi|\nabla \psi|_{2} \leq c|b|_{2}$ (weak bound for $\theta$ ). Taking a smooth family of mollifiers $\left\{\rho_{\eta}\right\}, \eta>0$, we obtain that $\psi \in H_{\Gamma_{1}}^{1}(\Omega) \cap H^{2}(\Omega)$. Indeed, let $\mathbf{u}_{\eta}:=\mathbf{u}_{\eta}^{\epsilon}+\mathbf{v}_{\eta}^{\epsilon}$, where $\mathbf{u}_{\eta}^{\epsilon}=\mathbf{u}^{\epsilon} * \rho_{\eta}, \mathbf{v}_{\eta}^{\epsilon}=\mathbf{v}^{\epsilon} * \rho_{\eta}$, and let $\psi_{\eta}$ be the solution in $H_{\Gamma_{1}}^{1}(\Omega)$ of the regularized system

$$
-\chi \Delta \psi_{\eta}=b+\left(\mathbf{u}_{\eta}^{\epsilon} \cdot \nabla\right) \psi_{\eta}+\left(\mathbf{v}_{\eta}^{\epsilon} \cdot \nabla\right) \psi_{\eta}:=F_{\eta},\left.\quad \psi_{\eta}\right|_{\Gamma_{1}}=0,\left.\quad \partial_{\mathbf{n}} \psi_{\eta}\right|_{\Gamma_{2}}=0 .
$$

Since $\mathbf{u}_{\eta}^{\epsilon}, \mathbf{v}_{\eta}^{\epsilon} \in C(\bar{\Omega})$ and $\nabla \psi_{\eta} \in \mathbf{L}^{2}(\Omega)$, we have $F_{\eta} \in L^{2}(\Omega)$. Now, we use Lemma 2.2 below in order to obtain $\psi_{\eta} \in H^{2}(\Omega)$ such that $\chi\left\|\psi_{\eta}\right\|_{2} \leq c\left|F_{\eta}\right|_{2}$, for some constant $c=c(\Omega)$. Using interpolation 
inequalities, the weak bound for $\theta$, and the Poincaré's inequality, we get $\left\|\psi_{\eta}\right\|_{2} \leq c \chi^{-1}\left(1+\chi^{-2}\left\|\mathbf{u}_{\eta}^{\epsilon}\right\|_{1}^{2}\right)|b|_{2}$. Taking the limit for a subsequence of $\{\eta\}$ and using the uniqueness of solution of $(4)$ in $H_{\Gamma_{1}}^{1}(\Omega)$, we get

$$
\|\psi\|_{2} \leq c \chi^{-1}\left(1+\chi^{-2}\left\|\mathbf{u}^{\epsilon}\right\|_{1}^{2}\right)|b|_{2}
$$

Finally, we consider the map that transforms $b \in L^{2}(\Omega)$ into the unique solution $\psi$ of (4) which is in $H^{2}(\Omega)$. This mapping is linear. By $(6)$, it is continuous from $L^{2}(\Omega)$ into $H^{2}(\Omega)$. Thus

$$
\mathcal{L}(b)=(h, \psi)+\chi<\zeta, \psi>_{H^{-1 / 2}\left(\Gamma_{2}\right), H^{1 / 2}\left(\Gamma_{2}\right)}-\chi \int_{\Gamma_{1}} \xi \partial_{\mathbf{n}} \psi d s,
$$

defines a continuous linear function of $b$ acting on $L^{2}$. We conclude from the Riesz representation that there exists a unique $\theta \in L^{2}(\Omega)$ such that $\mathcal{L}(b)=(\theta, b)$ for all $b \in L^{2}(\Omega)$. This proves the Lemma.

Lemma 2.2 Let $\Omega$ be either CASE 1 or CASE 2, and let $\psi \in H_{\Gamma_{1}}^{1}(\Omega)$ be the weak solution of the problem $-\Delta \psi=f$ in $\Omega$ with $\psi=0$ on $\Gamma_{1}$ and $\partial_{\mathbf{n}} \psi=0$ on $\Gamma_{2}$, for some data $f \in L^{2}(\Omega)$. Then, $\psi \in H^{2}(\Omega)$.

The result for domains of CASE 1 is a particular case of Theorem 1 in $[3,4]$. The result for domains of CASE 2 follows using analogous arguments to those in Theorem 4, IV-II of [10].

An estimate for $\theta$. Setting $b=\theta$ in the equation $(\theta, b)=\mathcal{L}(b)$, we obtain

$$
|\theta|_{2}^{2}=(h, \psi)+\chi<\zeta, \psi>_{H^{-1 / 2}\left(\Gamma_{2}\right), H^{1 / 2}\left(\Gamma_{2}\right)}-\chi \int_{\Gamma_{1}} \xi \partial_{\mathbf{n}} \psi d s,
$$

where $\psi \in H^{2} \cap H_{\Gamma_{1}}^{1}$ satisfying $\left.\partial_{\mathbf{n}} \psi\right|_{\Gamma_{2}} \equiv 0$ is the (unique) solution of (4) with $b=\theta$. Using the weak bound of $\theta$ for $b=\theta$, and (6), we obtain

$$
|\theta|_{2} \leq c \chi^{-1}\left\{\left(\chi^{-1}|h|_{2}+\left(1+\chi^{-1}\left\|\mathbf{u}^{\epsilon}\right\|_{1}\right)\left(|\xi|_{L^{2}\left(\Gamma_{1}\right)}+|\zeta|_{H^{-1 / 2}\left(\Gamma_{2}\right)}\right)\right\}\right.
$$

\section{Problem in $u$ and a related problem}

Problem 2. Consider $\theta \in L^{2}(\Omega), \mathbf{g} \in \mathbf{L}^{2}(\Gamma)$ and $\mathbf{f} \in \mathbf{L}^{3}(\Omega)$. Find $\mathbf{u} \in \mathbf{L}^{3}(\Omega)$ such that $(3)_{1,2}$ are satisfied. Now, we consider solenoidal test functions in $(3)_{1}$.

Lemma 3.1 Let $\Omega$ satisfy the hypothesis of Lemma 2.2. Consider the following boundary value problem for the Navier-Stokes equations with data $\mathbf{g} \in \mathbf{L}^{2}(\Gamma)$ satisfying $\int_{\Gamma} \mathbf{g} \cdot \mathbf{n} d s=0$ :

$$
-\nu \Delta \mathbf{z}+(\mathbf{z} \cdot \nabla) \mathbf{z}+\nabla p=0, \quad \nabla \cdot \mathbf{z}=0 \text { in } \Omega, \quad \mathbf{z}=\mathbf{g} \text { on } \Gamma .
$$

If $|\mathbf{g}|_{L^{2}(\Gamma)}$ is small enough, then there exists a unique weak solution $\mathbf{z} \in \mathbf{L}^{3}(\Omega)$ of problem (10). Moreover, there is a constant $c_{1}$ depending only on $\nu$ such that $|\mathbf{z}|_{3}<c_{1} \nu|\mathbf{g}|_{L^{2}(\Gamma)}\left(\nu-c_{1}|\mathbf{g}|_{L^{2}(\Gamma)}\right)^{-1}$.

PROOF. [Lemma 3.1] If $\Omega$ is a domain of CASE 2, the proof follows from Theorem 4 in [9]. If $\Omega \subset \mathbb{R}^{3}$ is a lipschitz bounded domain of CASE 1, we need to modify Lemma 2 in [9] (cf. remark 6 in [9]) in order to guarantee the strong regularity results for the Stokes problem in irregular domains. To this aim, we use Theorem 9.20 due to M. Dauge in [5].

In order to find $\mathbf{u}$, we split it into a large regular part $\mathbf{u}^{\epsilon}$ and a small irregular part $\mathbf{v}^{\epsilon}$, i.e., $\mathbf{u}=\mathbf{u}^{\epsilon}+\mathbf{v}^{\epsilon}$, where $\mathbf{u}^{\epsilon} \in \mathbf{H}^{1}(\Omega), \nabla \cdot \mathbf{u}^{\epsilon}=0$ and $\left.\mathbf{u}^{\epsilon}\right|_{\Gamma}=\mathbf{g}^{\epsilon}$, and $\mathbf{v}^{\epsilon} \in \mathbf{L}^{3}(\Omega)$ is the very weak solution of problem (10) such that $\left.\mathbf{v}^{\epsilon}\right|_{\Gamma}=\mathbf{g}-\mathbf{g}^{\epsilon}$. We choose $\mathbf{g}^{\epsilon}$ (for instance in $\mathbf{H}^{1 / 2}(\Gamma)$ ) as an approximation of $\mathbf{g}$ in $\mathbf{L}^{2}(\Gamma)$ such 
that $\left.\left|\mathbf{g}-\mathbf{g}^{\epsilon}\right|_{L^{2}} \ll 1\right)$. By Lemma 3.1, we know that if $\left|\mathbf{g}-\mathbf{g}^{\epsilon}\right|_{L^{2}(\Gamma)}$ is small enough, then there exists a unique solution $\mathbf{v}^{\epsilon} \in \mathbf{L}^{3}(\Omega)$ such that $\left|\mathbf{v}^{\epsilon}\right|_{3}<c_{1} \nu\left|\mathbf{g}-\mathbf{g}^{\epsilon}\right|_{L^{2}(\Gamma)}\left(\nu-c_{1}\left|\mathbf{g}-\mathbf{g}^{\epsilon}\right|_{L^{2}(\Gamma)}\right)^{-1}$ where $c_{1}=c_{1}(\nu)$. On the search for $\mathbf{u}^{\epsilon} \in \mathbf{H}^{1}(\Omega)$, we first lift the boundary data $\mathbf{g}^{\epsilon}$ using a suitable extension $\widehat{\mathbf{g}}^{\epsilon}$ satisfying $\left|B\left(\mathbf{w}, \widehat{\mathbf{g}}^{\epsilon}, \mathbf{w}\right)\right| \leq \frac{1}{2} \nu\|\mathbf{w}\|^{2}$ for all $\mathbf{w} \in \mathbf{V}$ (see for example [6] or [7] for Lipschitz domains, or [8,13] for the regular case). Thus, for some $\mathbf{v} \in \mathbf{V}$, we rewrite $\mathbf{u}^{\epsilon}=\mathbf{v}+\widehat{\mathbf{g}^{\epsilon}}$, and $\mathbf{u}=\mathbf{u}^{\epsilon}+\mathbf{v}^{\epsilon}=\mathbf{v}+\widehat{\mathbf{g}}^{\epsilon}+\mathbf{v}^{\epsilon}=\mathbf{v}+\mathbf{V}^{\epsilon}$ where $\mathbf{V}^{\epsilon}:=\widehat{\mathbf{g}^{\epsilon}}+\mathbf{v}^{\epsilon} \in \mathbf{L}^{3}(\Omega)$ and $\left.\mathbf{V}^{\epsilon}\right|_{\Gamma}=\mathbf{g}$. In this way, we need to solve the following problem in $\mathbf{v}$ :

$$
\nu((\mathbf{v}, \Phi))=B(\mathbf{v}, \Phi, \mathbf{v})+\beta(\theta \mathbf{f}, \Phi)+L(\mathbf{v}, \Phi)+<F, \Phi>
$$

for all $\Phi \in \mathbf{W}^{2,3 / 2}(\Omega) \cap \mathbf{W}_{0}^{1,3 / 2}(\Omega)$ with $\nabla \cdot \Phi=0$. The bilinear form $L(\cdot, \cdot)$ is defined by $L(\mathbf{v}, \Phi)=$ $B\left(\mathbf{V}^{\epsilon}, \Phi, \mathbf{v}\right)+B\left(\mathbf{v}, \Phi, \mathbf{V}^{\epsilon}\right)$, and the linear form $F$ by $<F, \Phi>=-\nu\left(\left(\widehat{\mathbf{g}}^{\epsilon}, \Phi\right)\right)+B\left(\mathbf{v}^{\epsilon}, \Phi, \widehat{\mathbf{g}^{\epsilon}}\right)+B\left(\widehat{\mathbf{g}}^{\epsilon}, \Phi, \mathbf{V}^{\epsilon}\right)$. If $\mathbf{v}$ is a solution of problem (11), then it is also a variational solution, i.e., equation (11) is also valid for all $\Phi \in \mathbf{V}$. One can check that $L(\mathbf{v}, \Phi),\langle F, \Phi\rangle$ and $B(\mathbf{v}, \Phi, \mathbf{v})$ are continuous linear mappings in $\Phi$ with respect to the topology of $\mathbf{H}^{1}(\Omega)$. Therefore, if we prove that $\mathbf{v} \in \mathbf{V}$ is a solution of (11), then it follows that $\mathbf{u}=\mathbf{v}+\mathbf{V}^{\epsilon}$ is a very weak solution of Problem 2 .

\section{Existence theorem}

We shall show how to construct a map $A: \mathbf{V} \longrightarrow \mathbf{V}$ whose fixed point gives a very weak solution of (2). Having $\theta$ (the unique solution of Problem 1), we define $A(\mathbf{v}) \in \mathbf{V}$ by the relation

$$
E(A \mathbf{v}, \Phi)=B(\mathbf{v}, \Phi, \mathbf{v})+\beta(\theta \mathbf{f}, \Phi)+<F, \Phi>, \quad \forall \Phi \in \mathbf{V}
$$

where $E(\mathbf{v}, \Phi):=\nu((\mathbf{v}, \Phi))-L(\mathbf{v}, \Phi)$. The bilinear form $E(\cdot, \cdot)$ is continuous and coercive under our assumptions. For each $\theta \in L^{2}(\Omega)$ and $\mathbf{v} \in \mathbf{V}$, the right-hand side of (12) defines a linear and bounded functional in $\Phi$ on $\mathbf{V}$. Thus, by the Lax-Milgram Lemma, the mapping $A$ is well defined. We observed that each fixed point $\mathbf{v} \in \mathbf{V}$ of the map $A$ defines a pair $(\mathbf{u}, \theta)=\left(\mathbf{v}+\mathbf{V}^{\epsilon}, \theta\right)$, which is a very weak solution of (2). Using the De Rham's Lemma we show then that there exists a $p \in W^{-1,3}(\Omega)$ such that the triple $(\mathbf{u}, \theta, p)$ satisfies all conditions in Definition 1.1. The existence of a fixed point of $A$ follows from the Schauder's fixed point Theorem. To this aim, we prove the existence of a radius $R$ such that the ball $B_{R}(\mathbf{V})=\left\{\mathbf{v} \in \mathbf{V}:\|\mathbf{v}\|_{1} \leq R\right\}$ satisfies the hypothesis of this theorem. We strongly use estimate (9) and the choice of $R$ depending on the data $\chi, \nu, \mathbf{f}, \zeta, \xi$ verifying the hypothesis of Theorem 1.2.

\section{Continuous dependence}

In [12], the continuous dependence with respect to the boundary data for $\theta$ was considered, but not for the $\mathbf{u}$-boundary data. As long as the first one does not present any additional difficulty, here we focus on the dependence result on $\left|\mathbf{g}_{1}-\mathbf{g}_{1}^{\epsilon}\right|_{L^{2}(\Gamma)} \neq 0$, for fixed data for $\xi$, $\zeta$. We consider

$$
\nu\left(\left(\mathbf{v}_{i}, \Phi\right)\right)=B\left(\mathbf{v}_{i}, \Phi, \mathbf{v}_{i}\right)+\beta\left(\theta_{i} \mathbf{f}, \Phi\right)+L\left(\mathbf{v}_{i}, \Phi\right)+<\mathbf{F}_{i}, \Phi>, \quad i=1,2, \forall \Phi \in \mathbf{V},
$$

where $L\left(\mathbf{v}_{i}, \Phi\right)=B\left(\mathbf{V}_{i}^{\epsilon}, \Phi, \mathbf{v}_{i}\right)+B\left(\mathbf{v}_{i}, \Phi, \mathbf{V}_{i}^{\epsilon}\right)$ and $<\mathbf{F}_{i}, \Phi>=-\nu\left(\left(\widehat{\mathbf{g}}_{i}^{\epsilon}, \Phi\right)\right)+B\left(\widehat{\mathbf{g}}_{i}^{\epsilon}, \Phi, \mathbf{V}_{i}^{\epsilon}\right)+B\left(\mathbf{v}_{i}^{\epsilon}, \Phi, \widehat{\mathbf{g}}_{i}^{\epsilon}\right)$, for $\mathbf{V}_{i}^{\epsilon}=\widehat{\mathbf{g}}_{i}^{\epsilon}+\mathbf{v}_{i}^{\epsilon}$. Taking the difference between the cases $i=1$ and $i=2$, setting $\Phi=\mathbf{v}_{1}-\mathbf{v}_{2}$, and estimating in a suitable manner, we obtain

$$
\begin{aligned}
\nu\left\|\mathbf{v}_{1}-\mathbf{v}_{2}\right\|_{1} & \leq c\left\|\mathbf{v}_{1}\right\|_{1}\left\|\mathbf{v}_{1}-\mathbf{v}_{2}\right\|_{1}+c\left|\theta_{1}-\theta_{2}\right|_{2}|\mathbf{f}|_{3}+c\left(\left\|\mathbf{v}_{1}\right\|_{1}+\left\|\widehat{\mathbf{g}}_{1}^{\epsilon}\right\|_{1}\right)\left|\mathbf{v}_{1}^{\epsilon}-\mathbf{v}_{2}^{\epsilon}\right|_{3} \\
& +\left(\nu+c\left|\mathbf{V}_{2}^{\epsilon}\right|_{3}+\left\|\mathbf{v}_{1}\right\|_{1}+\left\|\widehat{\mathbf{g}}_{1}^{\epsilon}\right\|_{1}\right)\left|\widehat{\mathbf{g}}_{1}^{\epsilon}-\widehat{\mathbf{g}}_{2}^{\epsilon}\right|_{1}+c\left|\mathbf{v}_{2}^{\epsilon}\right|_{3}\left\|\mathbf{v}_{1}-\mathbf{v}_{2}\right\|_{1} .
\end{aligned}
$$


In order to bound $\left|\mathbf{v}_{1}^{\epsilon}-\mathbf{v}_{2}^{\epsilon}\right|_{3}$ by $\left|\left(\mathbf{g}_{1}-\mathbf{g}_{1}^{\epsilon}\right)-\left(\mathbf{g}_{2}-\mathbf{g}_{2}^{\epsilon}\right)\right|_{L^{2}(\Gamma)}$, one first proves:

$$
\left|\mathbf{v}_{1}^{\epsilon}-\mathbf{v}_{2}^{\epsilon}\right|_{3} \leq C\left(1+\left|\mathbf{v}_{1}^{\epsilon}\right|_{3}+\left|\mathbf{v}_{2}^{\epsilon}\right|_{3}\right)\left|\left(\mathbf{g}_{1}-\mathbf{g}_{1}^{\epsilon}\right)-\left(\mathbf{g}_{2}-\mathbf{g}_{2}^{\epsilon}\right)\right|_{L^{2}(\Gamma)}
$$

Thus, assuming that $\nu$ is large enough and using the smallness of $\left|\mathbf{v}_{2}^{\epsilon}\right|_{3}$ and that $\nu\left\|\mathbf{v}_{1}\right\|_{1} \leq M$ with $M=$ $M\left(|\mathbf{f}|_{3},\left|h_{1}\right|_{2},\left\|\widehat{\mathbf{g}}_{1}^{\epsilon}\right\|_{1},\left|\xi_{1}\right|_{L^{2}\left(\Gamma_{1}\right)},|\zeta|_{H^{-1 / 2}\left(\Gamma_{2}\right)}\right)$, we obtain the following estimate of continuous dependence:

$$
\begin{aligned}
\nu\left\|\mathbf{v}_{1}-\mathbf{v}_{2}\right\|_{1} & \leq c\left|\theta_{1}-\theta_{2}\right|_{2}|\mathbf{f}|_{3}+c\left(\left\|\mathbf{v}_{1}\right\|_{1}+\left\|\widehat{\mathbf{g}}_{1}^{\epsilon}\right\|_{1}\right)\left(1+\left|\mathbf{v}_{1}^{\epsilon}\right|_{3}+\left|\mathbf{v}_{2}^{\epsilon}\right|_{3}\right)\left|\left(\mathbf{g}_{1}-\mathbf{g}_{1}^{\epsilon}\right)-\left(\mathbf{g}_{2}-\mathbf{g}_{2}^{\epsilon}\right)\right|_{L^{2}(\Gamma)} \\
& +c\left(\nu+c\left|\mathbf{V}_{2}^{\epsilon}\right|_{3}+\left\|\mathbf{v}_{1}\right\|_{1}+\left\|\widehat{\mathbf{g}}_{1}^{\epsilon}\right\|_{1}\right)\left|\mathbf{g}_{1}^{\epsilon}-\mathbf{g}_{2}^{\epsilon}\right|_{H^{1 / 2}(\Gamma)} .
\end{aligned}
$$

To obtain the estimate for $\left|\theta_{1}-\theta_{2}\right|_{2}$, we use that $\left(\theta_{i}, b_{i}\right)=\mathcal{L}\left(b_{i}\right)$ with $\mathcal{L}(b)$ as $(7)$, and $b_{i}=L_{\mathbf{u}_{i}}\left(\psi_{i}\right)$ with $L_{\mathbf{u}}:=-\chi \Delta \psi+(\mathbf{u} \cdot \nabla) \psi$. First, putting $\xi_{1}=\xi_{2}$ and $\zeta_{1}=\zeta_{2}$, and taking $\left(\theta_{1}, b_{i}\right)-\left(\theta_{2}, b_{i}\right)$ with $b_{i}=\theta_{1}-\theta_{2}$

$$
\left|\theta_{1}-\theta_{2}\right|_{2}^{2}=\left(h_{1}, \psi_{1}\right)-\left(h_{2}, \psi_{2}\right)+\chi<\zeta_{1},\left(\psi_{1}-\psi_{2}\right)>_{H^{-1 / 2}\left(\Gamma_{2}\right), H^{1 / 2}\left(\Gamma_{2}\right)}-\chi \int_{\Gamma_{1}} \xi_{1} \partial_{\mathbf{n}}\left(\psi_{1}-\psi_{2}\right) .
$$

Suitable estimates allow us to obtain $\left|\theta_{1}-\theta_{2}\right|_{2} \leq c\left|h_{1}-h_{2}\right|_{2}+c(\nu)\left|\mathbf{f}_{1}-\mathbf{f}_{2}\right|_{3}$ provided $\nu$ large enough. Since $\left|\mathbf{u}_{1}-\mathbf{u}_{2}\right|_{3}=\left|\mathbf{v}_{1}-\mathbf{v}_{2}\right|_{3} \leq c\left\|\mathbf{v}_{1}-\mathbf{v}_{2}\right\|_{1}$, we find $\left|\mathbf{u}_{1}-\mathbf{u}_{2}\right|_{3}+\left|\theta_{1}-\theta_{2}\right|_{2} \leq c(\nu)\left(\left|h_{1}-h_{2}\right|_{2}+\left|\mathbf{f}_{1}-\mathbf{f}_{2}\right|_{3}\right)$. If $\xi_{1} \neq \xi_{2}$ and $\zeta_{1} \neq \zeta_{2}$, then the additional terms $\chi \int_{\Gamma_{1}}\left(\xi_{1}-\xi_{2}\right) \partial_{\mathbf{n}} \psi_{2}$ and $\chi<\zeta_{1}-\zeta_{2}, \psi_{2}>_{H^{-1 / 2}\left(\Gamma_{2}\right), H^{1}\left(\Gamma_{2}\right)}$ appearing in (14) can be estimated by $c\left|\xi_{1}-\xi_{2}\right|_{L^{2}\left(\Gamma_{1}\right)}\left|\theta_{1}-\theta_{2}\right|_{2}$ and $c\left|\zeta_{1}-\zeta_{2}\right|_{H^{-1 / 2}\left(\Gamma_{2}\right)}\left|\theta_{1}-\theta_{2}\right|_{2}$ respectively.

\section{Acknowledgements}

The authors want to express their sincere gratitude to M. Dauge, V. Girault, C. Amrouche and F. Guillén-González for useful discussions and bibliographic references about this work.

\section{References}

[1] S. Chandrasekhar, Hydrodynamic and hydromagnetic stability, New york: Dover.

[2] C. Conca, Stokes equations with non-smooth data, Rev. Mat. Apl. Univ. Chile. 10, 115-122 (1989).

[3] M. Dauge, Problèmes mixtes pour le laplacien dans les domaines polyédraux courbes, C. R. Acad. Sci. Paris, t. 309, Série I, p. 553-558, (1989).

[4] M. Dauge, Newmann and Mixed Problems on Curvilinear Polyedra, Int. Equat. Oper. Birkhauser Verlag, Th 15 (1992).

[5] M. Dauge, Stationary Stokes and Navier-Stokes Systems on two- or three-Dimensional Domains with corners. Part I: Linearized Equations, SIAM J. Math. Anal., 20 (1) (1989).

[6] G. Galdi, An introduction to the Mathematical Theory of he Navier-Stokes Equations. Vol. I, II Springer-Verlag, 1994.

[7] V. Girault \& P. A. Raviart, Finite Element Methods for the Navier-Stokes equations, Springer-Verlag, 1980.

[8] J. L. Lions, Quelques Mthodes de Rsolution des Problmes aux Limites Non Linaires, Dunod, Paris, 1969.

[9] E. Marusič-Paloka, Solvability of the Navier-Stokes system with $L^{2}$ boundary data, Appl. Math. Optim. 41 (2000) 365-375.

[10] V. P. Mikhailov, Partial Diferential Equations, Mir Publishers, 1978.

[11] H. Morimoto, On the existence and uniqueness of the stationary solution to the equations of natural convection, Tokyo J. Math. 14 (1991) 217-226.

[12] M. Santos, M.A. Rojas-Medar, M.D. Rojas-Medar On the existence and uniqueness of the stationary solution to equations of natural convection with boundary data in $L^{2}$, Proc. R. Soc. London. A (2003) 459, 609-621.

[13] R. Teman, Navier-Stokes Equations: Theory and Numerical Analysis, North Holland, Amsterdam, New York, 1977. 